\title{
Enterovirus typing by immune electronmicroscopy
}

\author{
T. W. LEE, B. MEGSON* and J. B. KURTZ \\ Virus and Public Health Laboratory, John Radcliffe Hospital, Oxford OX3 9DU and *Virus Reference Division, \\ Central Public Health Laboratory, Colindale Avenue, London NW9 5HT
}

\begin{abstract}
A simple method of typing enteroviruses by immune electronmicroscopy (IEM) is given. Forty-four of 50 picornavirus strains typed by both IEM and neutralisation in cell culture gave identical results. Four strains could not be typed by one or other method. Two rhinovirus isolates were untypable by both methods. There were no discrepant results. The IEM method is convenient and has considerable savings in time and reagents.
\end{abstract}

\section{Introduction}

Since it was realised that there were many enteric viruses similar to poliovirus but not associated with paralysis, an important epidemiological activity of the virus laboratory has been to type enterovirus isolates from both clinical and environmental sources. The classic method for typing enteroviruses is by some form of neutralisation test with a range of specific antisera and assaying for inhibition of viral growth in cell cultures. This method has shown the enterovirus genus to contain a large number of viruses that cause disease in man. These are classified into poliovirus (1$3)$, coxsackie A (1-24), coxsackie B (1-6), echovirus (1-34) and enteroviruses (68-71) [1]. Neutralisation tests usually take several days to complete and are expensive in both reagents and operator time. We have developed a typing method based on immune electronmicroscopy (IEM) and compared it with the widely used neutralisation method.

\section{Materials and methods}

\section{Isolates}

Fifty isolates of picornaviruses (48 enteroviruses and two rhinoviruses) were examined. Thirty-eight isolates, which had been received by the virus reference laboratory (VRL), Colindale, for typing, were initially typed by neutralisation, coded and then examined by IEM in Oxford. Twelve isolates from patients in the

Received 29 June 1995; revised version accepted 28 July 1995.

Corresponding author: J. B. Kurtz.
Oxford region with clinical illnesses were similarly examined, first by IEM in Oxford and then, under code, by neutralisation at the VRL. Operators were unaware of any previous results.

\section{Neutralisation}

The picornaviruses were tested against five composite pools [2] of neutralising antisera prepared in rhesus monkeys (Virus Reference Laboratory, CPHL, Colindale, London). Virus suspensions $(50 \mu l)$ containing $c$. 100 TCD50 of virus were mixed with $50 \mu$ of each of the five antisera pools in five wells of a 96-well microtitration plate. After incubation for $2 \mathrm{~h}$ at room temperature, $100 \mu \mathrm{l}$ of a suspension of the most susceptible cells for each virus type (either rhesus monkey kidney or human embryo fibroblasts - MRC5) were added to each well $\left(c .1 \times 10^{5} \mathrm{cell} / \mathrm{ml}\right.$ in cell culture maintenance medium). The microtitration plate was incubated at $37^{\circ} \mathrm{C}$ in a humidified atmosphere containing $\mathrm{CO}_{2} 5 \%$ and examined after 3 and 6 days for viral cytopathic effect (CPE). The well showing no CPE contained the specific antiserum. The virus type was then determined in the same manner but with individual type-specific antisera (VRL). All tests were done in duplicate.

\section{Immune electronmicroscopy}

Four formvar/carbon coated grids (Agar Scientific, Stansted, Essex) were floated on to 5- $\mu 1$ drops of soluble staphylococcal protein A (10 $\mu \mathrm{g} / \mathrm{ml}$; Sigma) on non-wettable polythene-coated filter paper (Benchkote, Whatman, Maidstone). At the same time and also on Benchkote, each of four 4- $\mu$ l volumes of virus- 
containing fluid from cell cultures showing widespread CPE was mixed with an equal volume of one of four rabbit antisera pools. After storage of the treated grids and virus/antisera mixtures for $20 \mathrm{~min}$ at room temperature in a moist chamber, the grids were washed in distilled water, drained and refloated on the virus/ antisera drops. Incubation in the moist chamber was continued at $37^{\circ} \mathrm{C}$ for $1 \mathrm{~h}$. The four grids were then washed again in distilled water, stained with methylamine tungstate [3], and examined at $\times 40000$ magnification in a transmission electronmicroscope for the presence of immune complexes.

The 50 viruses were tested against four pools of rabbit antisera, used as supplied by the Laboratory of Microbiological Reagents, CPHL, Colindale Avenue, London, i.e., without further dilution. The four pools used for the initial tests were: (1) polyvalent polio; (2) polyvalent coxsackie $B$; (3) coxsackie A9; and (4) a 'megapool' consisting of echovirus pools 1-5 which together contain 25 specific antisera. A virus that formed immune complexes with one of the pools was then tested against the individual type-specific antisera comprising that pool to determine its type. Individual antisera against echovirus types 4, 20, 21 and 28, which were not present in the echovirus pools, were also used to test viruses that failed to react with the four pools.

\section{Results}

The appearance of the virus particles by IEM is shown in Fig. 1. The typical appearance of scanty nonaggregated virus particles in the presence of heterologous antisera (Fig. 1A) and the virus immune complexes with immunoglobulin 'bridges', formed in the presence of homologous serum (Fig. 1B) are clearly distinguishable. Non-viral aggregates, probably lipoprotein (Fig. 1C), again distinguishable from the immune complexes, were also seen occasionally. Both neutralisation and IEM gave the same results for 44 of the 50 virus isolates. These were identified as poliovirus type 1 (three isolates), type 2 (three isolates) and type 3 (two isolates); coxsackie A9 (three isolates); coxsackie B1 (two isolates), B2 (three isolates), B3 and B4 (one isolate each), B5 (three isolates); echovirus type 3 (three isolates), type 6 (two isolates), type 7 (four isolates), type 18 (two isolates), type 22 (four isolates), and types $4,11,15,17,21,25,27,30$ (one isolate each). Three isolates could not be typed by IEM. In one case this was because antiserum to the type (echovirus 16) is not included in the echo pools nor was it available in the Oxford laboratory. The other two isolates failed to grow in cell culture in Oxford and did not provide sufficient virus for IEM. Neutralisation had shown these to be echovirus types 7 and 19. One strain could not be typed by neutralisation as it failed to grow in rhesus monkey kidney cell cultures but was identified by IEM as echovirus type 22 . The two rhinoviruses were both untypable by either method. There were no discrepant results.

\section{Discussion}

This report describes a rapid method for typing enteroviruses by IEM and compares this with a traditional neutralisation method. Although the panels of antisera used in the two methods were different, one raised in rabbits and the other in rhesus monkeys, there was complete agreement between the results from IEM and neutralisation.

After specimen culture and detection of CPE, neutralisation tests with cell cultures require an initial
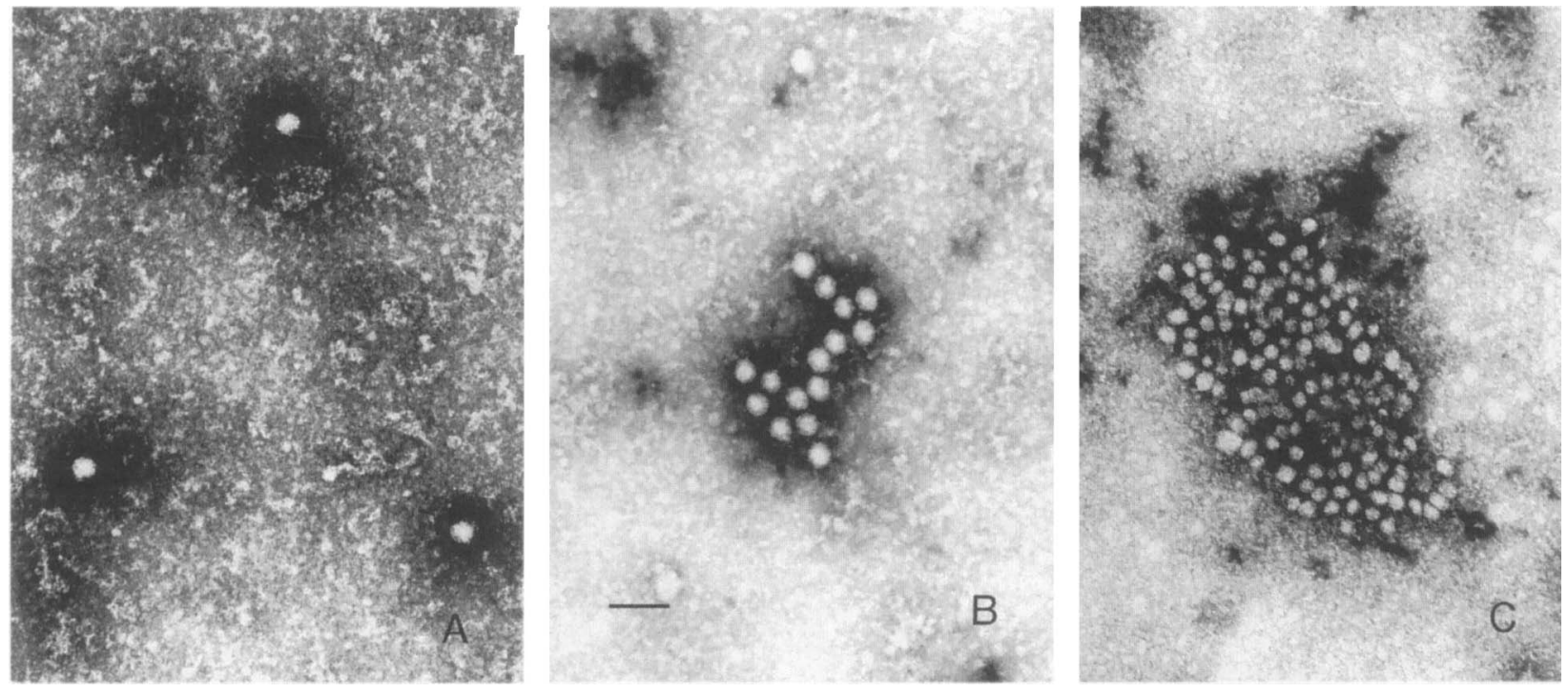

Fig. 1. Electronmicrographs of: A, unclumped enterovirus; B, immune-complexed enterovirus; C, lipoprotein aggregate. Methylamine tungstate stain, $b a r=100 \mathrm{~nm}$. 
titration of virus in order to determine the standard dose to be used with the panel of antisera. Following this, it usually takes at least 3 days before a CPE becomes apparent in the untreated control cells and hence virus neutralisation detected in antisera-treated cells. Specific type determination requires an additional 3 days. Therefore, it can often take 2 weeks to provide a result. These tests are also relatively expensive in reagents. Providing a laboratory has access to a transmission electronmicroscope, there are many benefits in using IEM to type viruses, with savings in both time and reagents as no dilution, titration or further culture of the virus is required. With IEM, complete enterovirus identification can be determined, from the initial specimen culture showing CPE in $4 \mathrm{~h}$, allowing for an initial screen and second IEM test with type-specific antisera. IEM uses less than one-tenth of the volume of antisera needed for a neutralisation test. Furthermore, the appearance of virus particles with IEM provides a confident result whereas slight viral growth often makes neutralisation in cell culture difficult to interpret. We have not encountered spontaneously aggregated viruses in IEM but it is advisable to examine all the grids in the primary screen. This should also enable mixed virus cultures to be detected.

A point of caution is that occasionally an antiserum may contain aggregates (probably lipoprotein, Fig. 1C), but the size of these particles is variable and none is empty, distinguishing them from virus immune complexes. Enterovirus typing by IEM provides a simple, rapid and cheaper alternative to the neutralisation method.

\section{References}

1. Committee on Enteroviruses. Classification of human enteroviruses. Virology 1962; 16: 501-504.

2. Hambling $\mathrm{MH}$, Davis PM, MacRae AD. The typing of enteroviruses in tissue culture by neutralization with composite antiserum pools. J Hyg 1963; 61: 479-484.

3. Faberge AC, Oliver RM. Methylamine tungstate, a new negative stain. J Microsc 1974; 20: 241-246. 